\title{
A pre-existing hydrophobic collapse in the unfolded state of an ultrafast folding protein
}

\author{
K. Hun Mok ${ }^{1,2}$, Lars T. Kuhn ${ }^{1}$, Martin Goez ${ }^{3}$, lain J. Day ${ }^{4, \dagger}$, Jasper C. Lin ${ }^{5}$, Niels H. \\ Andersen $^{5}$, and P. J. Hore ${ }^{1}$ \\ ${ }^{1}$ Department of Chemistry, University of Oxford, Physical \& Theoretical Chemistry Laboratory, \\ South Parks Road, Oxford OX1 3QZ, UK. ${ }^{2}$ School of Biochemistry and Immunology, Trinity \\ College, University of Dublin, Dublin 2, Ireland. ${ }^{3}$ Fachbereich Chemie, Martin-Luther-Universität \\ Halle-Wittenberg, Kurt-Mothes-Strasse 2, D-06120 Halle/Saale, Germany. ${ }^{4}$ Department of \\ Biochemistry and Molecular Pharmacology, University of Massachusetts Medical School, 364 \\ Plantation Street, Worcester, Massachusetts 01605, USA. ${ }^{5}$ Department of Chemistry, University \\ of Washington, Seattle, Washington 98195, USA.
}

\begin{abstract}
Insights into the conformational passage of a polypeptide chain across its free energy landscape have come from the judicious combination of experimental studies and computer simulations ${ }^{1,2}$. Even though some unfolded and partially folded proteins are now known to possess biological function $^{3}$ or to be involved in aggregation phenomena associated with disease states ${ }^{1,4}$, experimentally derived atomic-level information on these structures remains sparse as a result of conformational heterogeneity and dynamics. Here we present a technique that can provide such information. Using a 'Trp-cage' miniprotein known as TC5b (ref. 5), we report photochemically induced dynamic nuclear polarization $\mathrm{NMR}^{6}$ pulse-labelling experiments that involve rapid in situ protein refolding 7,8 . These experiments allow dipolar cross-relaxation with hyperpolarized aromatic side chain nuclei in the unfolded state to be identified and quantified in the resulting folded-state spectrum. We find that there is residual structure due to hydrophobic collapse in the unfolded state of this small protein, with strong inter-residue contacts between side chains that are relatively distant from one another in the native state. Prior structuring, even with the formation of non-native rather than native contacts, may be a feature associated with fast folding events in proteins.
\end{abstract}

Experimental advances in nuclear magnetic resonance (NMR) spectroscopy have led to the characterization of a diverse range of unfolded states of proteins ${ }^{9}$. In many cases the presence of residual structure has been shown ${ }^{10-13}$, but with some significant exceptions ${ }^{14}$ the poorly resolved spectra of the unfolded state, arising from conformational exchange and dynamic averaging, have generally hampered structural analysis by NMR. We report here the use of an NMR technique that circumvents some of these problems by transferring

\footnotetext{
Correspondence and requests for materials should be addressed to P.J.H. (peter.hore@chem.ox.ac.uk) and K.H.M. (mok1@tcd.ie).. Tresent address: Medway Sciences, University of Greenwich, Medway University Campus, Central Avenue, Chatham Maritime, Chatham, Kent, ME4 4TB, UK.

Author Contributions K.H.M. and I.J.D. built the in situ rapid mixing injector. K.H.M., M.G., I.J.D. and P.J.H. designed the experiments. K.H.M., L.T.K., M.G. and I.J.D. performed the experiments. J.C.L. and N.H.A. contributed the TC5b sample. M.G. developed the mathematical methods for obtaining NOE contact distances. K.H.M., L.T.K., M.G., I.J.D., N.H.A. and P.J.H. analysed the data. K.H.M., M.G., N.H.A. and P.J.H. wrote the paper. All authors discussed the results and commented on the manuscript.

Supplementary Information is linked to the online version of the paper at www.nature.com/nature.

The authors declare no competing financial interests.
} 
information from the unfolded or partially folded states of proteins to the native state ${ }^{15}$. Using this 'pulse-labelling' strategy, we probe the unfolded state of TC5b (relative molecular mass $M_{\mathrm{r}}=2,169$ ), a miniprotein that possesses key structural hallmarks normally only found in larger proteins ${ }^{5}$. Despite its high relative contact order, TC5b is an ultrafast folding protein ${ }^{16}$. This property, together with its small size, has made it an attractive subject for molecular dynamics folding simulations ${ }^{17-21}$.

Three methodologies are combined in this 'pulse-labelling' experiment (Fig. 1). (1) PhotoCIDNP (chemically induced dynamic nuclear polarization) ${ }^{6,22}$, a technique for enhancing the NMR signals ('hyperpolarization') of solvent-accessible tryptophan, tyrosine and histidine side chains by means of a laser-induced reaction of the protein with a flavin photosensitizer. (2) Rapid homogeneous mixing of solutions in the NMR sample tube to trigger the folding of a denatured protein on a timescale faster than nuclear spin-lattice relaxation (Supplementary Fig. 1) ) $^{7,8}$. To these two techniques we add here, for the first time, (3) transfer of nuclear magnetization via nuclear Overhauser effects (NOEs) from the hyperpolarized side chain protons to neighbouring atoms before the refolding step. As a result, inter-residue contacts in unfolded conformations can be detected in the well-resolved NMR spectrum of the refolded native state.

${ }^{1} \mathrm{H}$ photo-CIDNP measurements were initially performed on the native and denatured states of TC5b. The photo-CIDNP spectrum of native TC5b (Fig. 2b) is considerably simpler than the conventional NMR spectrum (Fig. 2a), because only two of the 20 side chains, Tyr 3 and Trp 6, are hyperpolarizable. The enhancements of the two aromatic side chains reflect the rates of their reaction with the photo-excited flavin 6,22 .

Figure $2 \mathrm{~b}-\mathrm{d}$ shows a series of photo-CIDNP spectra of native TC5b recorded with increasing laser irradiation times but keeping the delivered energy constant per flash. While the signal intensities from the directly polarized protons remain approximately constant, there are resonances in the aliphatic region (principally between 0.5 and 2.1 p.p.m.) that are essentially absent in Fig. $2 b$ but which grow in intensity as the irradiation time is increased. These are NOE peaks arising from transfer of polarization from Tyr 3 and Trp 6 via dipolar cross-relaxation ${ }^{23}$; they correlate well with the cross-peaks between protons separated by 2.0-3.5 ̊ previously observed in two-dimensional nuclear Overhauser effect spectroscopy (NOESY) spectra of the native state ${ }^{5}$ (Supplementary Fig. 2). The mixture of absorptive and emissive signals in the aliphatic regions of Fig. $2 \mathrm{c}$ and $\mathrm{d}$ reflect the different phases of the hyperpolarized tyrosine and tryptophan nuclei ${ }^{23}$.

In contrast, the NMR (Fig. 2e) and photo-CIDNP spectra of TC5b unfolded in $6.0 \mathrm{M}$ urea (Fig. 2f-h) differ noticeably from those of the native state. The polarized aromatic Trp 6 signals are stronger than those of Tyr 3; together with their linewidth differences and chemical shift movements, this suggests a less structured and more accessible environment for Tyr 3 in the denatured state. The NOESY spectra of the denatured state (Supplementary Fig. 2) show that almost all of the peaks have different chemical shifts. Furthermore, most of the amide and aromatic protons are not well-resolved in the spectrum of the denatured state, making direct resonance assignment extremely difficult. Nonetheless, the photo-CIDNP spectra at longer irradiation times (Fig. 2f-h) do display NOEs.

The pulse-labelling experiment (Fig. 1) entails production of photo-CIDNP and build-up of NOEs in the denatured state, followed by rapid injection of the protein solution into refolding buffer in the NMR sample tube. A free induction decay is acquired after a specified delay $(100 \mathrm{~ms})$ to allow complete mixing in situ ${ }^{7}$. The resulting CIDNP spectrum of the refolded native state exhibits aliphatic resonances whose amplitudes reflect the CIDNP-NOEs that were generated before folding. TC5b is well suited to this procedure, as 
refolding has been shown to be very rapid (the time constant for refolding being $4 \mu \mathrm{s}$; ref. 16).

The pulse-labelled ('Unfolded-to-Native', U-to-N) spectrum of TC5b (Fig. 3b) differs considerably from the conventional CIDNP spectrum of native TC5b (Figs $2 \mathrm{~d}$ and $3 \mathrm{~d}$ ), especially with regard to the prominent signals in the aliphatic region (0.5-2.0 p.p.m.). Control experiments in which conditions were chosen so that the injection resulted in no change of state of the protein were also performed: U-to-U and N-to-N (Fig. 3a and c, respectively). These control spectra strongly resemble the corresponding conventional CIDNP spectra (Fig. 2h and d, respectively), supporting the conclusion that the aliphatic signals in the U-to-N pulse-labelled spectrum truly reflect inter-residue contacts in the unfolded ensemble.

The U-to-N NOE peaks (Fig. 3b) can be identified straightforwardly from the NMR chemical shift assignments of the native state (Supplementary Fig. 2 and Supplementary Table 1). Four side chains were found to be recipients of cross-polarization: Ile 4, Leu 7, Pro 12 and Arg 16. In addition, because Tyr 3 is largely exposed to the solvent and wellremoved from neighbouring side chains (Supplementary Fig. 3), Trp 6 is likely to be the major source of the NOEs seen in the U-to-N spectrum. Average distances of the Ile 4, Leu 7, Pro 12 and Arg 16 protons from the Trp 6 side chain in the unfolded state can be estimated as detailed in the Supplementary Methods. Using experimentally determined ${ }^{1} \mathrm{H}$ spin-lattice relaxation times for native and unfolded states (Supplementary Table 2), estimates of the proton-proton distances are obtained using, as a calibration, the ensembleaveraged distance of $3.41 \AA$ from Trp 6 HD1 to the Arg 16 HB2 proton in the TC5b native state (Table 1).

The NOE contact distances observed between Trp 6 and Ile 4, Leu 7, Pro 12 and Arg 16 sites in the unfolded state are, with one exception, shorter than those in the compact native state (Table 1). Although the NOEs in the U-to-N spectra could be attributed to independent close contacts in different conformers rather than a more compact ensemble characterized by a hydrophobic collapse, this would require unrealistically short distances in individual conformers, particularly for the Trp 6/Pro 12 interaction. The hydrodynamic radius of the unfolded state, obtained from diffusion NMR experiments $(8.0 \pm 0.2 \AA$; Supplementary Fig. $4)$, is slightly larger than that of the native state $(7.4 \pm 0.1 \AA)$, but not nearly large enough to be consistent with a fully unfolded, random coil structure ${ }^{24}$. Thus, the short distances observed appear to result from simultaneously co-existing contacts within a compact ensemble of structures. Figure 4a shows a representation of a partially collapsed state consistent with the U-to-N NOE observations. A notable non-native interaction involves Ile 4 , which lies on the opposite side of the amino-terminal helix and hence is not within NOE range in the native state (Fig. 4b). In addition, although Arg 16 forms a stabilizing salt bridge with Asp 9 in the native state ${ }^{5}$, it is the aliphatic part of the Arg 16 side chain that is found to be associated with Trp 6 in the unfolded state. Furthermore, native-state NOEs between Trp 6 and Pro 17-19 are absent in the U-to-N spectrum. Instead, and in agreement with chemical shift melting data ${ }^{5}$, the unfolded state has a closer association of Trp 6 and Pro 12. Our conclusion regarding a pre-formed hydrophobic cluster in TC5b is consistent with spectroscopic results that show that the denatured state ensemble of the Ile4Gly substitution mutant contains a larger fraction of random coil structures ${ }^{25}$, and that Trp 6 is situated within a hydrophobic environment in the thermally-denatured state ${ }^{26}$.

What implications does the existence of structural features in the unfolded ensemble have for the fast folding of TC5b? The existence, in simulated folding trajectories, of the Asp 9/ Arg 16 salt bridging before achieving the native orientation of the Trp 6 side chain with respect to the prolines has been viewed either as a potential kinetic trap ${ }^{21}$ or as an 
accelerating feature ${ }^{18}$. In the case of a Pro12Trp variant designed to favour prior formation of an edge-to-face $\operatorname{Trp} 6 / \operatorname{Trp} 12$ interaction, Trp-cage folding is further accelerated ${ }^{27}$, suggesting that the close interaction between $\operatorname{Trp} 6$ and Pro 12 found here may contribute to the fast folding of the protein. In another case, fluorescence studies on TC5b analogues have shown that a decrease in folding speed is found when elements of the residual structure in the denatured state are removed ${ }^{25}$. Simulation studies with an extended starting structure suggest that there is an initial collapse event, with different hydrophobic interactions formed depending on the specific trajectory ${ }^{17,18}$; these interactions may determine the further course of the simulated folding. In agreement with some of the interactions found in silico ${ }^{18}$, we find that both native and non-native specific contacts pre-exist in the unfolded ensemble. Hence, it appears that the pre-formation of hydrophobically collapsed structures in the equilibrated unfolded state may be a significant factor in accelerating folding ${ }^{28}$.

The CIDNP pulse-labelling technique can be applied generally to other proteins, provided that a suitable aromatic side chain is present and refolding is fast enough to compete with the relaxation of the nuclear hyperpolarization. Applications to proteins that fold more slowly than $\sim 100 \mathrm{~ms}$ will require either more dramatic hyperpolarizations ${ }^{22,29}$ or slower nuclear spin-lattice relaxation ${ }^{30}$. We therefore anticipate that this approach will provide valuable information on the structures of denatured states of many proteins, particularly those that refold rapidly.

\section{METHODS}

\section{In situ rapid mixing injection device}

The in situ rapid mixing injection device recently developed and used for real-time protein refolding $\mathrm{NMR}^{7}$ and CIDNP pulse-labelling $\mathrm{NMR}^{8}$ experiments was further modified to improve its performance and durability. Details of the proteins and reagents and the injection device can be found in Supplementary Information and Supplementary Fig. 1.

\section{Rapid refolding and NMR experiments}

A volume of $30 \mu \mathrm{l}$ of 1.5-2.0 mM TC5b or ${ }^{19}$ F-Tyr-TC5b (a control, see Supplementary Information) in $6.0 \mathrm{M}$ urea, $0.05 \mathrm{M}$ sodium citrate buffer, $\mathrm{pH} 7.5$, with $0.3 \mathrm{mM}$ FMN was loaded as injectant. The experiment was performed at $5{ }^{\circ} \mathrm{C}$ to ensure essentially complete refolding to the native state ${ }^{5}$ (Supplementary Fig. 5). Polarization of the injectant was carried out using an $\mathrm{Ar}^{+}$laser (Spectra Physics BeamLok 2080, multiline mode, $24 \mathrm{~W}$ output power) gated for a specified time period (usually $500 \mathrm{~ms}$ ) using a mechanical shutter (NM Laser Products LS200). The unfolded protein solution was then pneumatically injected into $300 \mu \mathrm{l}$ of refolding buffer $(0.05 \mathrm{M}$ sodium citrate, $\mathrm{pH}$ 7.5) contained in the NMR sample tube, resulting in an 11-fold dilution of the denaturant. Complete mixing and folding is accomplished within $50 \mathrm{~ms}$ of injection ${ }^{7}$. (The first-order time constant for refolding of TC $5 \mathrm{~b}$ has been determined ${ }^{16}$ to be $4 \mu \mathrm{s}$.) Each pulse-labelled spectrum was the difference between the sum of eight 'light' and 'dark' experiments, where 'light' spectra are those acquired after a laser flash, and 'dark' spectra are those acquired without laser illumination. Further details on the photo-CIDNP and conventional NMR experiments, along with data analysis and extraction of cross-polarized distances, are described in Supplementary Information.

\section{Supplementary Material}

Refer to Web version on PubMed Central for supplementary material. 


\section{Acknowledgments}

We thank I. Kuprov for the synthesis of 9-fluorenylmethoxycarbonyl-O-t-butyl-3-fluoro-L-Tyr and for NMR experiments on the ${ }^{19}$ F-Tyr-TC5b variant; T. Nagashima, C.J.V. Jones and H. Paisley for help with the conceptual design, building and testing of the rapid mixing injector; R. Gerber for assistance in acquiring the NMR spectra; A.L. Davis for discussions and for providing spectrometer time for the diffusion measurements; L.J. Smith, C. Redfield, A.E. Mark and D.A.C. Beck for valuable discussions; and S. Min for assistance in figure preparation. K.H.M. also thanks M. Nilges, R. Wade and the EMBO Practical Course on Biomolecular Simulation. The authors are indebted to C.M. Dobson for continued encouragement in the application of photo-CIDNP to protein folding problems. This work was supported by the BBSRC (K.H.M., L.T.K., and P.J.H.), the Studienstiftung des deutschen Volkes (L.T.K.), the Deutsche Forschungsgemeinschaft (M.G.), and the US National Institutes of Health (N.H.A. and J.C.L.).

\section{References}

1. Dobson CM. Protein folding and misfolding. Nature. 2003; 426:884-890. [PubMed: 14685248]

2. Fersht AR, Daggett V. Protein folding and unfolding at atomic resolution. Cell. 2002; 108:573-582. [PubMed: 11909527]

3. Dyson HJ, Wright PE. Intrinsically unstructured proteins and their functions. Nature Rev. Mol. Cell Biol. 2005; 6:197-208. [PubMed: 15738986]

4. Chiti F, Dobson CM. Protein misfolding, functional amyloid, and human disease. Annu. Rev. Biochem. 2006; 75:333-366. [PubMed: 16756495]

5. Neidigh JW, Fesinmeyer RM, Andersen NH. Designing a 20-residue protein. Nature Struct. Biol. 2002; 9:425-430. [PubMed: 11979279]

6. Kaptein, R. Biological Magnetic Resonance. Berliner, LJ.; Reuben, J., editors. Plenum; New York: 1982. p. 145-191.

7. Mok KH, et al. A rapid sample-mixing technique for transient NMR and Photo-CIDNP spectroscopy: Applications to real-time protein folding. J. Am. Chem. Soc. 2003; 125:1248412492. [PubMed: 14531692]

8. Mok KH, Nagashima T, Day IJ, Hore PJ, Dobson CM. Multiple subsets of side-chain packing in partially folded states of a-lactalbumins. Proc. Natl Acad. Sci. USA. 2005; 102:8899-8904. [PubMed: 15956205]

9. Dyson HJ, Wright PE. Unfolded proteins and protein folding studied by NMR. Chem. Rev. 2004; 104:3607-3622. [PubMed: 15303830]

10. Klein-Seetharaman J, et al. Long-range interactions within a nonnative protein. Science. 2002; 295:1719-1722. [PubMed: 11872841]

11. Shortle D, Ackerman MS. Persistence of native-like topology in a denatured protein in $8 \mathrm{M}$ urea. Science. 2001; 293:487-489. [PubMed: 11463915]

12. Yi Q, Scalley-Kim ML, Alm EJ, Baker D. NMR characterization of residual structure in the denatured state of protein L. J. Mol. Biol. 2000; 299:1341-1351. [PubMed: 10873457]

13. Crowhurst KA, Forman-Kay JD. Aromatic and methyl NOEs highlight hydrophobic clustering in the unfolded state of an SH3 domain. Biochemistry. 2003; 42:8687-8695. [PubMed: 12873128]

14. Religa TL, Markson JS, Mayor U, Freund SMV, Fersht AR. Solution structure of a protein denatured state and folding intermediate. Nature. 2005; 437:1053-1056. [PubMed: 16222301]

15. Balbach J, et al. Detection of residue contacts in a protein folding intermediate. Proc. Natl Acad. Sci. USA. 1997; 94:7182-7185. [PubMed: 9207065]

16. Qiu L, Pabit SA, Roitberg AE, Hagen SJ. Smaller and faster: The 20-residue Trp-cage protein folds in 4 ms. J. Am. Chem. Soc. 2002; 124:12952-12953. [PubMed: 12405814]

17. Simmerling C, Strockbine B, Roitberg AE. All-atom structure prediction and folding simulations of a stable protein. J. Am. Chem. Soc. 2002; 124:11258-11259. [PubMed: 12236726]

18. Snow CD, Zagrovic B, Pande VS. The Trp cage: Folding kinetics and unfolded state topology via molecular dynamics simulations. J. Am. Chem. Soc. 2002; 124:14548-14549. [PubMed: 12465960]

19. Chowdhury S, Lee MC, Xiong G, Duan Y. Ab initio folding simulation of the Trp-cage miniprotein approaches NMR resolution. J. Mol. Biol. 2003; 327:711-717. [PubMed: 12634063] 
20. Pitera JW, Swope W. Understanding folding and design: Replica-exchange simulations of "Trpcage" miniproteins. Proc. Natl Acad. Sci. USA. 2003; 100:7587-7592. [PubMed: 12808142]

21. Zhou R. Trp-cage: Folding free energy landscape in explicit water. Proc. Natl Acad. Sci. USA. 2003; 100:13280-13285. [PubMed: 14581616]

22. Mok KH, Hore PJ. Photo-CIDNP NMR methods for studying protein folding. Methods. 2004; 34:75-87. [PubMed: 15283917]

23. Hore PJ, Egmond MR, Edzes HT, Kaptein R. Cross-relaxation effects in the photo-CIDNP spectra of amino acids in proteins. J. Magn. Reson. 1982; 49:122-150.

24. Kohn JE, et al. Random-coil behavior and the dimensions of chemically unfolded proteins. Proc. Natl Acad. Sci. USA. 2004; 101:12491-12496. [PubMed: 15314214]

25. Neuweiler H, Doose S, Sauer M. A microscopic view of miniprotein folding: Enhanced folding efficiency through formation of an intermediate. Proc. Natl Acad. Sci. USA. 2005; 102:1665016655. [PubMed: 16269542]

26. Ahmed Z, Beta IA, Mikhonin AV, Asher SA. UV-resonance Raman thermal unfolding study of Trp-cage shows that it is not a simple two-state miniprotein. J. Am. Chem. Soc. 2005; 127:1094310950. [PubMed: 16076200]

27. Bunagan MR, Yang X, Saven JG, Gai F. Ultrafast folding of a computationally designed Trp-cage mutant: Trp ${ }^{2}$-cage. J. Phys. Chem. B. 2006; 110:3759-3763. [PubMed: 16494434]

28. Sánchez IE, Kiefhaber T. Hammond behavior versus ground state effects in protein folding: Evidence for narrow free energy barriers and residual structure in unfolded states. J. Mol. Biol. 2003; 327:867-884. [PubMed: 12654269]

29. Khan F, Kuprov I, Craggs TD, Hore PJ, Jackson SE. ${ }^{19}$ F NMR studies of the native and denatured states of green fluorescent protein. J. Am. Chem. Soc. 2006; 128:10729-10737. [PubMed: 16910667]

30. Lyon CE, Jones JA, Redfield C, Dobson CM, Hore PJ. Two-dimensional ${ }^{15} \mathrm{~N}-{ }^{1} \mathrm{H}$ photo-CIDNP as a surface probe of native and partially structured proteins. J. Am. Chem. Soc. 1999; 121:65056506. 


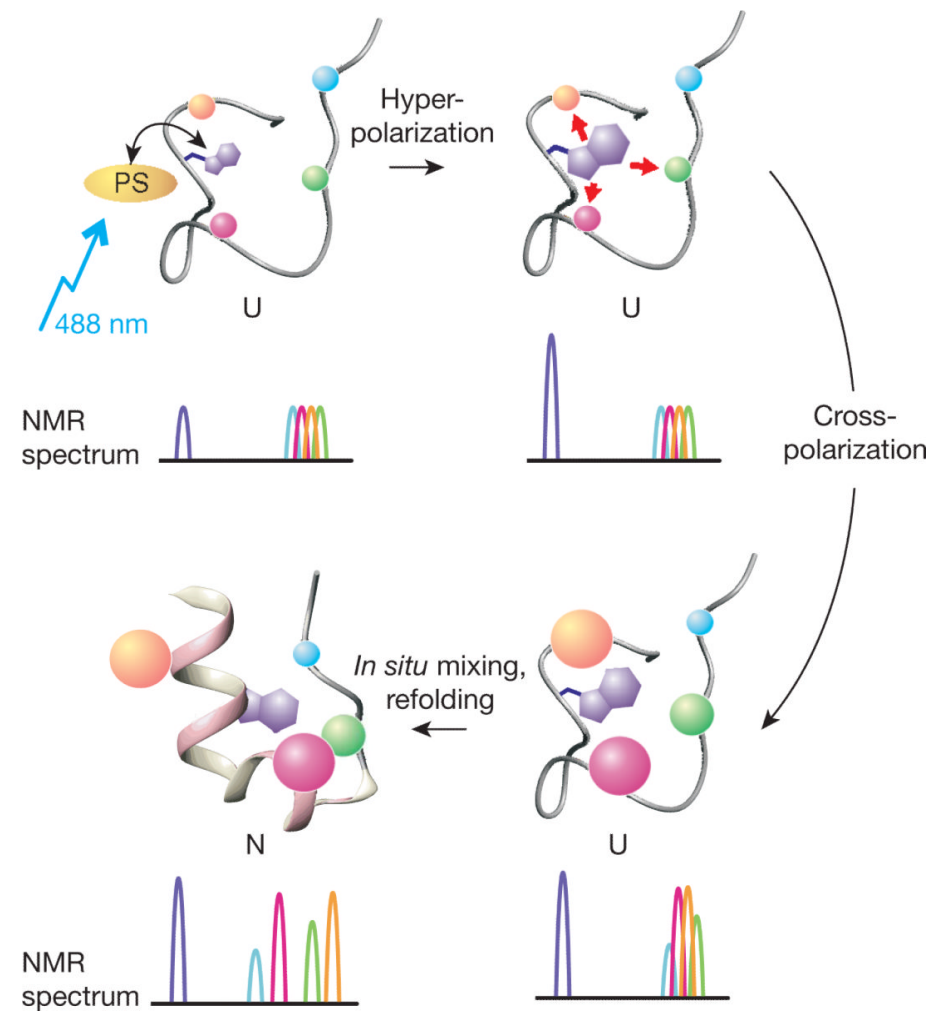

Figure 1. Diagram of the photo-CIDNP pulse labelling technique

Laser excitation of a freely-diffusing photosensitizing molecule and its subsequent interaction with a solvent-accessible aromatic amino-acid side chain (violet) results in a nonBoltzmann nuclear spin state, which further transfers its polarization via NOEs to neighbouring side chains (coloured spheres). For unfolded or partially folded proteins, detection is carried out after refolding the protein to its native state, where the greater chemical shift dispersion in the NMR spectrum allows identification of individual atoms. The increase in the size of spheres represents the extent of cross-polarization. PS, photosensitizer, $\mathrm{U}$, unfolded state; $\mathrm{N}$, native (folded) state. 


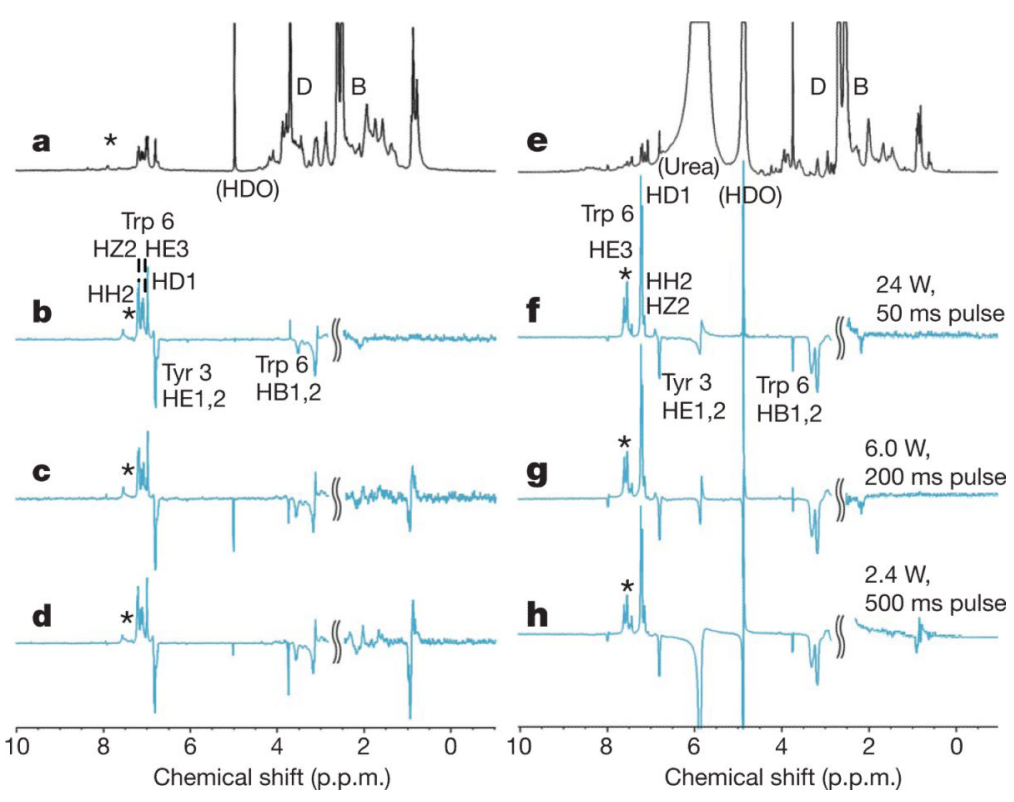

Figure 2. The $600 \mathrm{MHz}{ }^{1} \mathrm{H}$ NMR and photo-CIDNP spectra of TC5b

$\mathbf{a}-\mathbf{d}$, In its native state; $\mathbf{e}-\mathbf{h}$, in its denatured state. a, e, ${ }^{1} \mathrm{H}$ NMR spectra for the native state (a) and the $6.0 \mathrm{M}$ urea-denatured state (e) of TC5b at $5{ }^{\circ} \mathrm{C}$. For photo-CIDNP spectra b-d and $\mathbf{f}-\mathbf{h}$, the power and duration of the laser irradiation were varied as shown. The aliphatic region is magnified four times to aid visualization. Chemical shift assignments of the hyperpolarized side chains of Tyr 3 and Trp 6 are given, together with those of water (HDO), the chemical shift reference (1,4-dioxane, D), the buffer (citrate, B) and urea. In the native state spectra $\mathbf{c}$ and $\mathbf{d}$, the strong emissive cross-polarized peaks of Ile 4 HG2, HD1 and Leu 7 HD1 ( 0.9 p.p.m.) arise from the proximity of the Tyr 3 HE2 protons in the native state. Likewise, the absorptive signals from Pro $12 \mathrm{HG}$ ( 2.08 p.p.m.) and Leu $7 \mathrm{HG}$ (1.6 p.p.m.) reflect NOEs from Trp 6. Additional small signals (labelled with asterisks) arise from minor conformer populations associated with cis isomers of the four proline residues, which all have trans conformations in the completely folded native state and in any conformations that can rapidly achieve the folded state. 


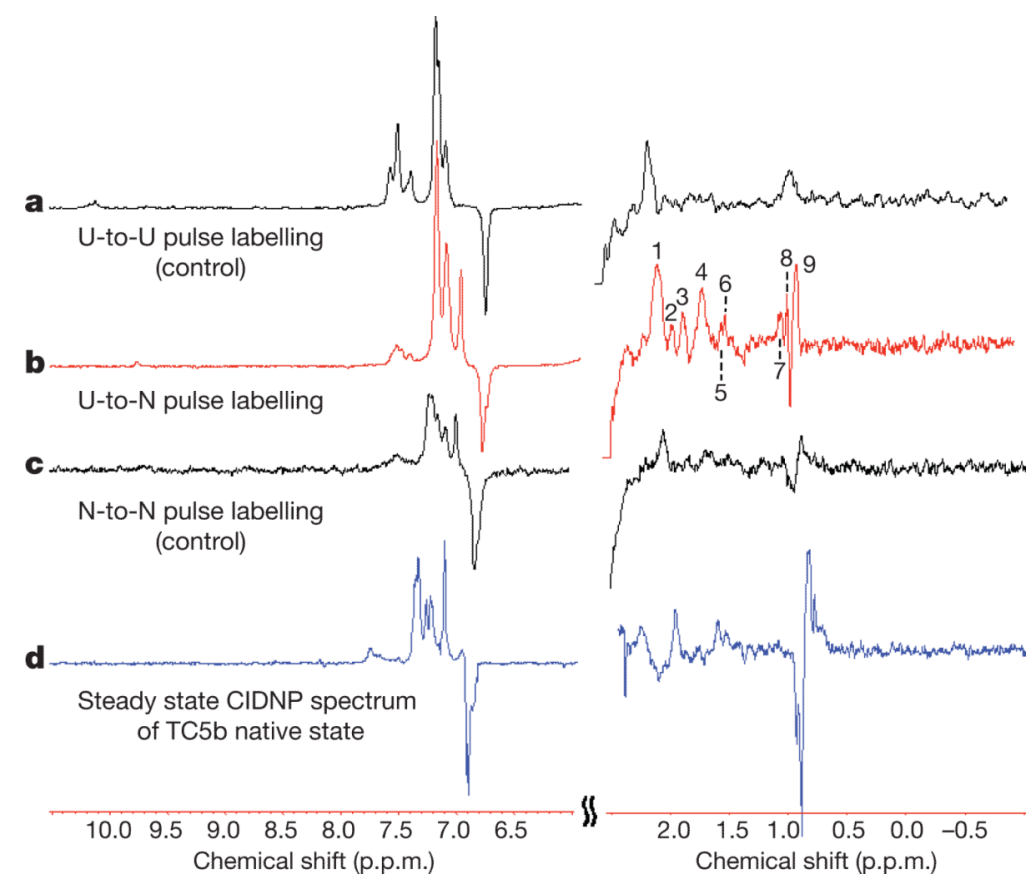

Figure 3. The $600 \mathrm{MHz}$ photo-CIDNP pulse-labelled NMR spectra of TC5b

The laser irradiation time was $500 \mathrm{~ms}$ at a power of $5.0 \mathrm{~W}$. a, Pulse-labelled control spectrum of the unfolded state of TC5b in $6.0 \mathrm{M}$ urea (' $\mathrm{U}$-to-U pulse labelling'). b, Pulselabelled spectrum of the unfolded state of TC5b ('U-to-N pulse labelling'). c, Pulse-labelled control spectrum of the native state of TC5b in $0.55 \mathrm{M}$ urea ('N-to-N pulse labelling'). d, Steady-state CIDNP spectrum of the native state of TC5b (identical to Fig. 2d). The assignment of peaks in the aliphatic region is as follows: 1, Pro 12 HB2 and HG2; 2, Ile 4 HB; 3, Arg 16 HB2; 4, Arg 16 HB3; 5, Leu 7 HG; 6, Ile 4 HG2; 7, Leu 7 HD1; 8, Ile 4 HG2, HD1; and 9, Leu 7 HD2. 


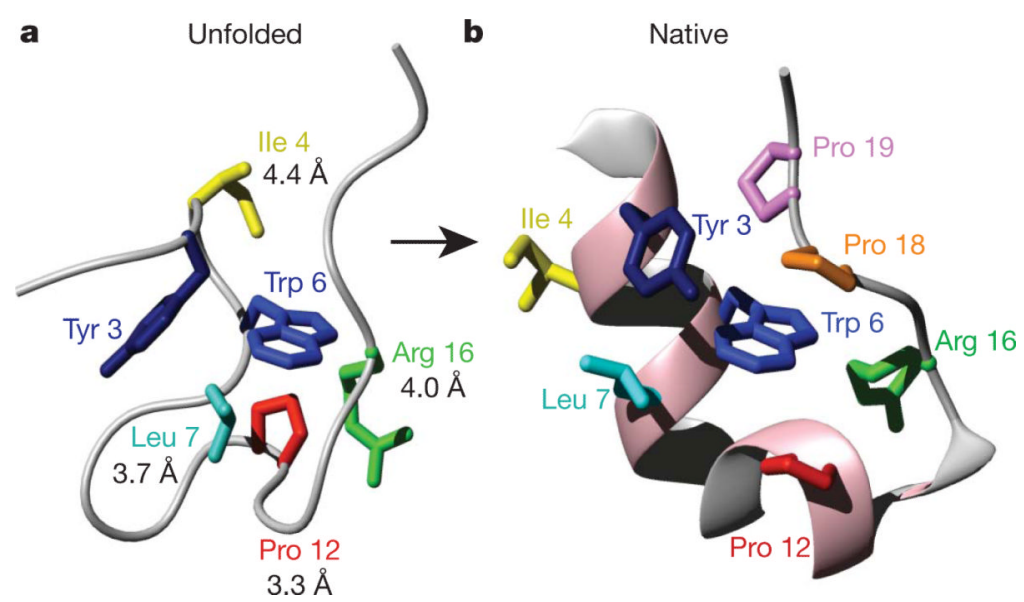

Figure 4. Representation of the three-dimensional structures of TC5b

a, Unfolded state; $\mathbf{b}$, native state. Cross-polarized side chains identified in the pulse-labelled spectrum (Ile 4, Leu 7, Pro 12, Arg 16) are indicated in a, and the approximate mean distances from the cross-polarization source (Trp 6) are shown. The ribbon structure of the denatured state in a is purely schematic; however, structural features are generally drawn according to experimental results given in this and other works (see text for citations). 


\section{Table 1}

Estimated distances of cross-polarized nuclei from $\operatorname{Trp} 6$

\begin{tabular}{lllllllll}
\hline & \multicolumn{8}{c}{ Cross-polarized protons } \\
\cline { 2 - 8 } & Ile 4 & Ile 4 & Leu 7 & Leu 7 & Pro 12 & Arg 16 & Arg16 \\
& HB & HG2 & HD1 & HD2 & HG2 & HB2 & HB3 \\
& & HD1 & & & & & \\
\hline Unfolded state $(\AA)$ & 4.2 & 4.5 & 4.0 & 3.4 & 3.3 & 4.2 & 3.8 \\
Native state $(\AA)$ & $8.07^{\dagger}$ & $8.71^{\dagger}$ & $4.81^{\dagger}$ & $3.73^{\dagger}$ & $4.02^{\dagger}$ & $3.24^{\S}$ & $4.68^{\S}$ \\
& & $8.75^{\dagger}$ & & & & & \\
\hline
\end{tabular}

Distances observed by pulse-labelled photo-CIDNP of TC5b.

* The data for the native state are ensemble-averaged distances to the nearest hyperpolarized aromatic proton of Trp 6 in the NMR structure (ref. 5)

${ }^{\dagger}$ Nearest hyperpolarized Trp 6 proton HE3

${ }_{\mathrm{HH} 2}$

$\S_{\mathrm{HE} 1 .}$ 